\title{
Der Phosphatstoffwechsel des Facettenauges im Dunkeln und im Licht
}

\author{
Helmut LANGer \\ Zoologisches Institut der Universität München
}

\begin{abstract}
The phosphate metabolism of the compound eye in darkness and in light. The phosphate metabolism of the compound eye of the blow-fly (males of wild-type and mutant chalky [LANGER 1962b] of Calliphora erythrocepbala MEIGEN) was investigated in connection with its function as a sensory organ. Microchemical analyses were made to determine changes in the amounts of phosphorus compounds. After having kept flies in darkness or under illumination with ultraviolet light for six hours, the substances of the isolated eyes were separated according to SCHNEIDER (1945). In no fraction did the phosphate content vary between darkadapted and illuminated eyes. On the other hand, the amount of free inorganic phosphate increased with illumination, whereas the phosphate liberated from acid-instable compounds during 7 minutes hydrolysis $\left(\mathrm{N} \mathrm{HCl} ; 100^{\circ} \mathrm{C}\right)$ decreased correspondingly. This effect was more pronounced in mutant chalky than in wild-type eyes. Specific enzymatic analyses of the adenine nucleotides showed - already after two hours - significantly less ATP in illuminated than in dark-adapted eyes of mutant chalky. In order to check the influence of adequate stimulation on the turnover of phosphate compounds, the extent of incorporation of radioactive phosphorus in the eyes was measured after feeding carrier free $32 \mathrm{P}$-orthophosphate to starved flies $(1 \mu \mathrm{C}$ per fly). One to four hours after feeding, the over-all incorporation was found to be $35 \%$ higher in light than in darkness $(P<0.01)$. Within the fractions phosphatides showed an increase nearly three times that of the acid soluble compounds. This means that independently of the general rise in metabolic rate, there is a specific effect of illumination. Phosphatides are assumed to have special importance for the processes of excitation in the light sensitive cells of the compound eye.
\end{abstract}

\section{EINLEITUNG}

Das Facettenauge der Insekten stellt ein besonders günstiges Objekt dar, um den Stoffwechsel eines Sinnesgewebes im Zusammenhang mit seiner Receptorfunktion biochemisch zu untersuchen. Das Gewebe besteht nur aus wenigen Zelltypen; den größten Teil seiner Masse stellen die Sinneszellen dar. In einem solchen Gewebe kann man Veränderungen in den Sinneszellen auch dann noch chemisch-analytisch finden, wenn sie nur relativ klein sind. Dies stellt einen Vorteil gegenüber der Retina von Wirbeltieren dar, die neben den Sinneszellen sehr viel nervöse Anteile enthält.

Seit einiger Zeit haben wir uns mit dem Stoffwechsel des Facettenauges beschäftigt (LANGer 1960a, b) und zuletzt den Kohlenhydrat-Stoffwechsel im Zusammenhang mit der Funktion untersucht (LANGer 1962a). Bei diesen Versuchen wird stets eine Gruppe von belichteten Augen mit einer Kontrollgruppe verglichen, die gleichzeitig für 
dieselbe Zeitspanne im Dunkeln gehalten wird. Die Belichtung kann sowohl mit sichtbarem als auch mit ultraviolettem Licht erfolgen, da beide für das Insektenauge einen adäquaten Reiz darstellen. Hier wurde die Belichtung mit ultraviolettem Licht vorgenommen, um Temperatureffekte auszuschließen. (Messungen innerhalb der Retina ergaben, daß deren Temperatur um nicht mehr als $\pm 1^{\circ} \mathrm{C}$ von der Temperatur der Ungebung und damit der dunkel gehaltenen Kontrolltiere abweicht). In den belichteten Augen findet sich stets eine geringere Menge von Gesamtkohlenhydrat als in den dunkel gehaltenen. Aus dem vermehrten Kohlenhydratverbrauch läßt sich der Energiebedarf berechnen, der für den Sehvorgang in einer bestimmten Zeit nötig ist; er beträgt bei der verwendeten Beleuchtungsstärke in einem Auge etwa 0,01 cal pro Stunde (LANGER 1962a).

In engem Zusammenhang mit dem Kohlenhydrat-Stoffwechsel steht der Stoffwechsel der Phosphorsäure, über den hier berichtet werden soll. Von der Retina der Wirbeltiere ist bekannt, daß in vitro bei Belichtung o-Phosphorsäure freigesetzt wird (LANGE \& SIMON 1922). Es ist anzunehmen, daß diese aus dem ATP stammt, das bei der Funktion verbraucht und von den Glykolyse-Prozessen nicht in genügender Menge nachgebildet wird. Über den Phosphatstoffwechsel der Wirbeltierretina sind in den letzten Jahren, vor allem von japanischen Autoren (Honjo \& KATo 1952, 1953, 1955, SEKoGUTI 1953, Akrya 1956), eine Anzahl von Ergebnissen erarbeitet worden. Beim Facettenauge liegen die Verhältnisse insofern etwas anders, als unter normalen Bedingungen in vitro keine Glykolyse vorkommt (LANGER 1962a), während anzunehmen ist, daß zumindest bei den Säugetieren die aerobe Glykolyse in der Retina ein physiologischer Vorgang ist (Warburg et al. 1924, Cohen \& Noell 1960). Im Facettenauge muß bei der Funktion eine hohe Rate von oxydativer Phosphorylierung erfolgen.

Für die Untersuchung des Phosphatstofiwechsels bestand also zunächst die Frage, $o b$ bei Belichtung eine Mengenveränderung in den an der oxydativen Phosphorylierung beteiligten Metaboliten eintritt oder ob eine Anderung in deren Umsatzrate erfolgt. Daneben wurde bei den Substanzen des sog. Baustoffwechsels untersucht, $a b$ Mengen- oder, was wahrscheinlicher war, Umsatzgrößenveränderungen im Zusammenhang mit der Funktion aufzufinden sind.

\section{MATERIAL UND METHODEN}

Das Objekt der Untersuchungen ist das Auge der Schmeißfliege Calliphora erythrocephala Mergen. Für die Versuche wurden Männchen überwiegend in der 2. und 3. Woche des Imaginallebens verwendet, und zwar neben denen der normalen rotäugigen Wildform auch solche von einem mutierten Stamm ohne Schutzpigmente in den Augen. Die Augen der Mutante, chalky (c) (LANGer 1962b), enthalten weder Farbstoffe der Ommochrom- nodh der Pteridin-Gruppe, besitzen aber das eigentliche Sehpigment (LANGER \& PATAT 1962). Diese Tiere können normal sehen, wie aus Verhaltensbeobachtungen und auch aus elektrophysiologischen Versuchen (HoFfmanN \& LANGER 1961) hervorgeht. Die Belichtung der Fliegen erfolgte mit einem QuecksilberBrenner ('Typ NN 15/44VK, „Hanau“), der neben wenig blauem vorwiegend ultraviolettes Licht ( $\mathrm{Hg}$-Linien zwischen 254 und $436 \mathrm{~nm}$ ) abgibt; die Tiere befanden sich 
über weiß glasiertem Porzellan-Untergrund in 8 bis $9 \mathrm{~cm}$ Abstand von der Lichtquelle. An den herauspräparierten Augen wurden chemische Aufarbeitungen vorgenommen; die Fraktionierung erfolgte nach SCHNEIDER (1945) in fünf Stoffgruppen mit Hilfe von Säure und Lauge sowie Lipoid-Lösungsmitteln. Für die Subfraktionierung innerhalb der leicht säurelöslichen Verbindungen wurden Perchlorsäure-Extrakte verwendet und darin folgende Anteile bestimmt: Das freie anorganische Phosphat nach MARTIN \& Dotr (1949) direkt und nach 7 Minuten Hydrolyse in 1 in Salzsäure bei $100^{\circ} \mathrm{C}$ (Lohmann 1928), die Adenin-Nucleotide mit enzymatischer Methodik im optischen Test nach Warburg (Bergmeyer 1962).

\section{UNTERSUCHUNG DER MENGEN DER PHOSPHATVERBINDUNGEN}

Die Ergebnisse für eine ganze Anzahl von Analysen des Phosphatgehaltes an unbehandelten Tieren sind als Mittelwerte in Tabelle 1 zusammengestellt. Bei den Hauptfraktionen fällt auf, daß die Phosphatide einen relativ hohen Anteil am Gesamtphosphat ausmachen. Innerhalb der säurelöslichen Fraktion wird von dem organisch gebundenen Phosphat ein erheblicher Anteil, etwa die Hälfte, durch die Hydrolyse freigesetzt. Das ATP macht von diesem Material nur etwa 35 bis $40 \%$ aus. Es muß

\section{Tabelle 1}

Vcrteilung der Phosphorverbindungen auf die Fraktionen des Materials unbehandelter Augen. Mittelwerte in nMol P pro 1 Auge von männlichen Tieren

\begin{tabular}{|lccc|}
\hline Fraktion & $\begin{array}{c}\text { Anzahl } \\
\text { der Bestimmungen }\end{array}$ & nMol P/Auge & $\begin{array}{c}\text { Anteil am } \\
\text { Gesamtphosphor } \\
(\% / 0)\end{array}$ \\
\hline Leicht säurelösliche Verbindungen & 21 & 21,07 & 34,4 \\
Freies anorganisches Phosphat & 28 & 3,81 & \\
Freies Phosphat nach & 15 & 10,46 & \\
7 Minuten Hydrolyse & 4 & 2,73 & \\
ATP & 5 & 0,58 & \\
ADP & 6 & 1,97 & 42,5 \\
AMP & 19 & 25,97 & 17,9 \\
Phosphatide & 12 & 10,94 & 4,3 \\
Nucleinsäuren & 23 & 2,65 & 0,9 \\
Phosphoproteide & 22 & 0,55 & \\
Rest (Chitin) & & & \\
\hline
\end{tabular}

also noch eine größere Menge von anderen säurelabilen Phosphatverbindungen vorhanden sein. Auffallend ist die molare Verteilung der Adenin-Nucleotide mit dem hohen Prozentsatz an AMP; durch Kontrollen konnte sichergestellt werden, daß es sich dabei nicht um ein durch die Präparation der Augen bedingtes Artefakt handelt.

In der ersten Versuchsgruppe wurden die Augen von normalen, rotäugigen Fliegen verglichen, die 6 Stunden bestrahlt oder gleichzeitig ebenso lange im Dunkeln gehalten worden waren. Tabelle 2 zeigt die Ergebnisse. In den Hauptfraktionen der Aufarbeitung finden sich keinerlei Veränderungen der Mengen. Dagegen zeigt das freie anorganische Phosphat nach Bestrahlung eine Zunahme um ungefähr 20\%, die 
Tabelle 2

Phosphorgehalt in den Fraktionen des Augenmaterials nad 6 Std. Dunkelhaltung oder Belichtung. Mittelwerte aus 6 Versuchen an Tieren der Wildform in nMol P pro 1 Auge

\begin{tabular}{|lccc|}
\hline Fraktion & $\begin{array}{c}\text { nach } \\
\text { Dunkelhaltung }\end{array}$ & $\begin{array}{c}\text { nach } \\
\text { Belichtung }\end{array}$ & $\begin{array}{c}\text { Differenz } \\
\text { (\%) von } \\
\text { Dunkelaugen) }\end{array}$ \\
\hline Leicht säurelösliche Verbindungen & 20,4 & 20,4 & \pm 0 \\
Freies anorganisches Phosphat & 5,5 & 6,6 & +20 \\
Freies Phosphat nach & 11,8 & 12,2 & +3 \\
7 Minuten Hydrolyse & 26,7 & 27,5 & +3 \\
Phosphatide & 12,2 & 12,3 & +1 \\
Nucleinsäuren & 3,1 & 3,0 & -3 \\
Phosphoproteide + Rest & & & \\
\hline
\end{tabular}

beim freien Phosphat nach Hydrolyse nicht auftritt. Es ist daher anzunehmen, daß energiereiche Phosphatverbindungen im Zusammenhang mit der Funktion gespalten werden.

Daraufhin wurde eine Versuchsserie durchgeführt, in der die Veränderungen des Gehaltes an freiem anorganischem Phosphat bei verschieden langer Belichtungszeit in den Augen von normalen und von chalky-Fliegen bestimmt wurden. In Abbildung 1 ist die Differenz im Gehalt an freiem anorganischem Phosphat von belichteten und dunkelgehaltenen Augen gegen die Versuchszeit aufgetragen. Jeder Punkt stellt das Mittel aus 5 bis 6 Versuchen dar; die Differenzen sind - mit Ausnahme des Punktes für 2 Stunden Belichtung an normaläugigen Fliegen - statistisch gut gesichert $(P<0,01)$. Es ergibt sich also eine etwa lineare Zunahme des freigesetzten Phosphates mit der Belichtungszeit, der Anstieg ist bei der pigmentfreien Mutante $c$ signifikant steiler als

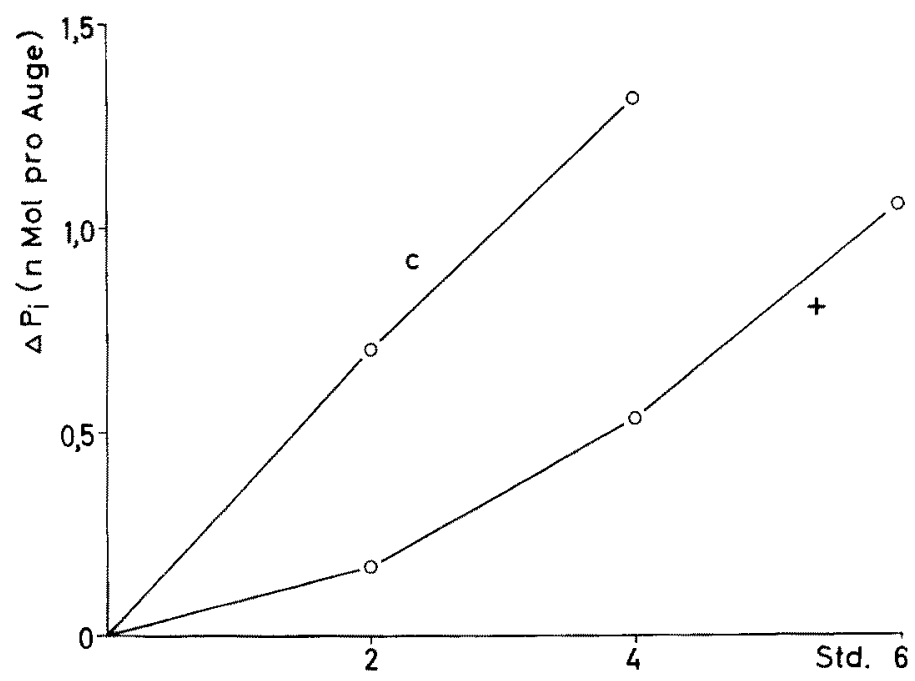

Abb. 1: Zunahme des freien anorganischen Phosphats in Augen von Tieren der Normalform und der Mutante $c$ bei verschiedener Dauer der Belichtung. Dargestellt ist die Differenz $\left(\Delta \mathrm{P}_{\mathrm{i}}\right)$ zwischen den Werten für belichtete und für gleichzeitig dunkelgehaltene Augen in nMol pro 1 Auge über die Zeit. Mittelwerte aus 5 bis 6 Versuchen pro Kurvenpunkt 
Tabelle 3

Gehalt an Adeninnucleotiden und freiem anorganischem Phosphat in Augenmaterial nach 2 Std. Dunkelhaltung oder Belichtung. Mittelwerte aus 4 bis 6 Versuchen an Tieren der Mutante chalky in nMol pro 1 Auge

\begin{tabular}{|c|c|c|c|c|c|c|}
\hline & $\begin{array}{l}\text { nach Dur } \\
\text { nMol/ } \\
\text { Auge }\end{array}$ & $\begin{array}{l}\text { kelhaltung } \\
\text { o von } \\
\text { Summe }\end{array}$ & $\begin{array}{l}\text { nach I } \\
\text { nMol/ } \\
\text { Auge }\end{array}$ & $\begin{array}{l}\text { lichtung } \\
\% \% \text { von } \\
\text { Summe }\end{array}$ & $\begin{array}{l}\text { Di } \\
\text { nMol/ } \\
\text { Auge }\end{array}$ & $\begin{array}{l}\text { erenz } \\
\quad \% \text { yon } \\
\text { Dunkelaugen }\end{array}$ \\
\hline \multicolumn{7}{|l|}{ Adenin-Nucleotide: } \\
\hline Summe & 5,28 & & 4,54 & & $-0,74$ & -14 \\
\hline ATP & 2,73 & 52 & 2,02 & 45 & $-0,71$ & -26 \\
\hline ADP & 0,58 & 11 & 0,78 & 17 & $+0,20$ & +35 \\
\hline AMP & 1,97 & 37 & 1,74 & 38 & $-0,23$ & -12 \\
\hline \multicolumn{7}{|l|}{ Freies anorganisches } \\
\hline Phosphat & 3,95 & & 4,65 & & $+0,70$ & +18 \\
\hline
\end{tabular}

bei der Wildform ( $\mathrm{P}<0,01$ im $t$-Test für die Anstiegssteilheiten). In einem Teil der Versuche wurde auch der Gehalt an freiem Phosphat nach 7 Minuten Hydrolyse bestimmt. Er verändert sich nur wenig und nicht in erkennbarem Zusammenhang mit der Belichtungszeit.

Daneben wurde an der Mutante $c$ nach 2 Stunden Dunkelheit oder Belichtung der Gehalt an Adenin-Nucleotiden direkt bestimmt. Tabelle 3 zeigt die Ergebnisse für den Gehalt an ATP, ADP und AMP, zusammen mit dem des freien anorganischen Phosphats, als Mittel aus 4 bis 6 Versuchen berechnet. Nach Belichtung findet sich eine geringfügig, aber signifikant verminderte Menge an Nucleotiden. Das ATP wird prozentual weit stärker vermindert als die Gesamtmenge, während das ADP sowohl relativ als auch absolut etwas zunimmt. Dagegen ist die Verminderung des AMP nicht größer als die der Gesamtmenge, so daß sein Anteil praktisch unverändert bleibt. Alle Unterschiede sind, ebenso wie beim freien anorganischen Phosphat, mit $\mathrm{P} \leqq 0,01$ statitisch gut gesichert. Damit ist gezeigt, daß der Gehalt an energiereichem Phosphat bei der Funktion der Augen geringer wird.

\section{UNTERSUCHUNG DERUMSATZRATEN DER PHOSPHATVERBINDUNGEN}

Um den Einfluß der Funktion der Augen auf den Umsatz der Phosphatverbindungen zu ermitteln, wurde der Einbau von radioaktivem Phosphat in die Substanzen der Augen bestimmt. Dazu wurde jeweils $1 \mu \mathrm{C}$ trägerfreies ${ }^{32 \mathrm{P}-O r t h o p h o s p h a t ~ i n ~ k o n-~}$ zentrierter Rohrzudkerlösung zu einem gegebenen Zeitpunkt an hungrige Fliegen individuell verfüttert; nach einer bestimmten Zeit erfolgte die Präparation der Augen.

Als Vorversuch war es nötig, den Zeitverlauf des Einbaues zu bestimmen; dafür wurden Fliegen in diffusem Tageslicht verwendet. Der Gesamteinbau in die Augen zeigt einen Anstieg bis etwa 4 Stunden nach der Fütterung und dann einen langsamen Abfall. Der zeitliche Verlauf des Einbaues in die Haupt-Fraktionen ist in Abbildung 2 dargestellt. Der Verlauf der Kurve für die leicht säurelöslichen Verbindungen ist nahezu der gleiche wie der für den Gesamteinbau, da sich 80 bis $95 \%$ des Gesamteinbaues in dieser Fraktion wiederfinden. Der Einbau in die übrigen Fraktionen geht viel lang- 


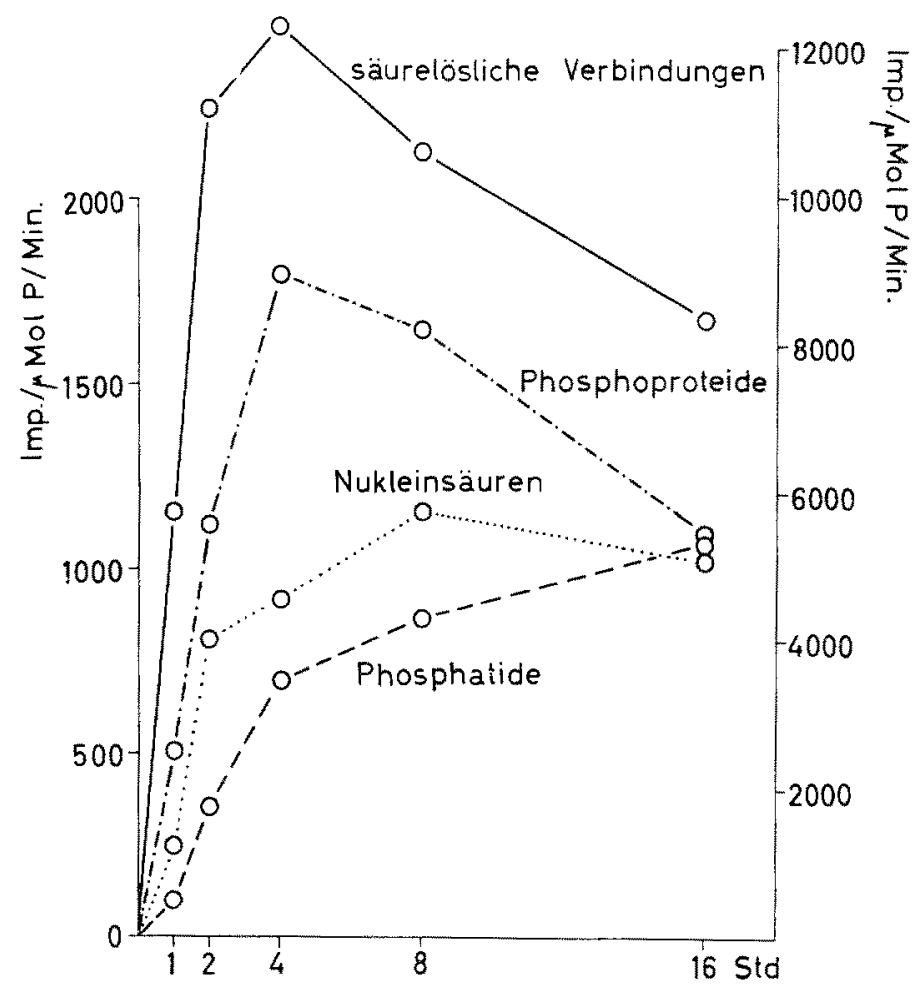

Abb. 2: Zeitlicher Verlauf des Einbaues von ${ }^{32} \mathrm{PO}_{4^{--}}$in die Haupt-Fraktionen des Augenmaterials von Normaltieren nach einmaliger Fütterung. Aufgetragen ist die spezifische Aktivität der Phosphatverbindungen über die Zeit; die rechte Ordinate mit 5 fach verkleinertem Maßstab gilt nur für die leicht säurelöslichen Verbindungen. Mittelwerte aus je 3 Versuchen

samer vor sich, am langsamsten der in die Phosphatide, wo selbst nach 16 Stunden noch kein Maximum der spezifischen Aktivität erreicht ist.

Um die Bestrahlungsversuche durchzuführen, wurde nur der Zeitraum verwendet, in dem alle Fraktionen noch eine ansteigende spezifische Aktivität zeigen, also bis 4 Stunden nach Fütterung des radioaktiven Materials. Die Versuchsanordnung mit dem Vergleich von dunkelgehaltenen und bestrahlten Fliegen aus ein und derselben Zucht war die gleiche wie für die vorher dargestellten Untersuchungen. Tabelle 4 zeigt die Ergebnisse für ein typisches Experiment an normaläugigen Fliegen mit einer Versuchszeit yon 4 Stunden. Die spezifische Aktivität der Substanzen aller Fraktionen ist nach Belichtung höher als nach Dunkelhaltung; der Anstieg beträgt für den Gesamteinbau $35 \%$. Jedoch ist diese Zunahme des Einbaues durchaus nicht in allen Fraktionen gleich groß. Die stärkste Steigerung findet sich bei den Phosphatiden.

Derartige Versuche wurden mit 1,2 und 4 Stunden Dauer mehrfach an normalen und an chalky-Fliegen durchgeführt; da sich ein Unterschied zwischen den beiden Augenfarbtypen dabei bisher nicht feststellen ließ, werden die Ergebnisse zusammengefaßs. In Tabelle 5 sind die Mittelwerte aus den 18 Versuchen dieser Serie zusammengestellt. Die Zahlen sind Prozentwerte für die spezifische Aktivität der 
Tabelle 4

Größe des ${ }^{32}$ P-Einbaues in das Material der Augen nach 4 Std. Dunkelhaltung oder Belichtung. Ergebnisse eines typischen Versuches an Tieren der Wildform in Imp./Min/ $/ \mathrm{Mol} \mathrm{P}$

\begin{tabular}{|lccc|}
\hline Fraktion & $\begin{array}{c}\text { nach } \\
\text { Dunkelhaltung }\end{array}$ & $\begin{array}{c}\text { nach } \\
\text { Belichtung }\end{array}$ & $\begin{array}{c}\text { Differenz } \\
\text { (\%/ovon } \\
\text { Dunkelaugen) }\end{array}$ \\
\hline Gesamtmaterial & 8520 & 11470 & +35 \\
Leidht säurelösliche Verbindungen & 22100 & 28580 & +29 \\
Phosphatide & 1140 & 2330 & +104 \\
Nucleinsäuren & 1530 & 2320 & +52 \\
Phosphoproteide & 3540 & 5320 & +50 \\
\hline
\end{tabular}

\section{Tabelle 5}

Zunahme der spezifischen Aktivität in den Fraktionen des Augenmaterials bei Belichtung. Mittlere Prozentwerte für belichtete Augen aus 6 Versuchen für jeden Zeitpunkt an Tieren der Wildform und der Mutante chalky; $100 \%$ ist jeweils die spezifische Aktivität der entsprechenden Fraktionen aus gleichzeitig untersuchten Augen dunkelgehaltener Tiere

\begin{tabular}{|c|c|c|c|}
\hline Versuchsdauer & 1 Stunde & 2 Stunden & 4 Stunden \\
\hline Leicht säurelösliche Verbindungen & 145 & 139 & 115 \\
\hline Phosphatide & 210 & 189 & 164 \\
\hline Nucleinsäuren & 167 & 168 & 127 \\
\hline Phosphoproteide & 183 & 172 & 133 \\
\hline
\end{tabular}

Phosphatverbindungen aus den bestrahiten Augen, bezogen auf die entsprechenden Werte aus den gleichzeitig dunkel gehaltenen Augen, die jeweils als 100\% gesetzt werden. Sie stellen also die Zunahme auf Grund der Lichteinwirkung dar.

Die gefundene Steigerung durch Belichtung wird mit längerer Versuchsdauer in allen Fraktionen geringer, wie dies wegen des Ausgleichs im Einbau zu erwarten ist. Für jeden Zeitpunkt liegen die Größen der Erhöhungen in den Fraktionen in der gleichen Reihenfolge; die geringste Zunahme findet sich bei den säurelöslichen Verbindungen, die die Intermediärprodukte für den Einbau in die anderen Substanzgruppen enthalten, die stärkste bei den Phosphatiden. Nur für diese ist die Zunahme statistisch gesichert stärker als für die säurelöslichen Verbindungen $(P<0,01)$, während die Steigerungen bei Nucleinsäuren und bei Phosphoproteiden von der der säurelöslichen Verbindungen nicht signifikant verschieden sind.

Die gegenüber den anderen Substanzen nahezu dreimal größere Erhöhung der Umsatzrate im Licht gibt einen Hinweis auf einen spezifischen Effekt der Belichtung auf die Phosphatide. Um von der möglicherweise unspezifischen Erhöhung des durchschnittlichen Einbaues unabhängig zu werden, wird das Verhältnis der spezifischen Aktivitäten des Phosphates in den Fraktionen der leicht säurelöslichen Verbindungen und der Phosphatide berechnet. Diese Verhältniszahlen sind in Abbildung 3 für dunkelgehaltene und für belichtete Augen über der Zeit aufgetragen. Sie werden erwartungsgemäß mit längeren Zeiten immer niedriger, sind aber in jedem Falle für Augen im Licht kleiner als für solche im Dunkeln, und zwar immer in der Relation 0,7:1. 


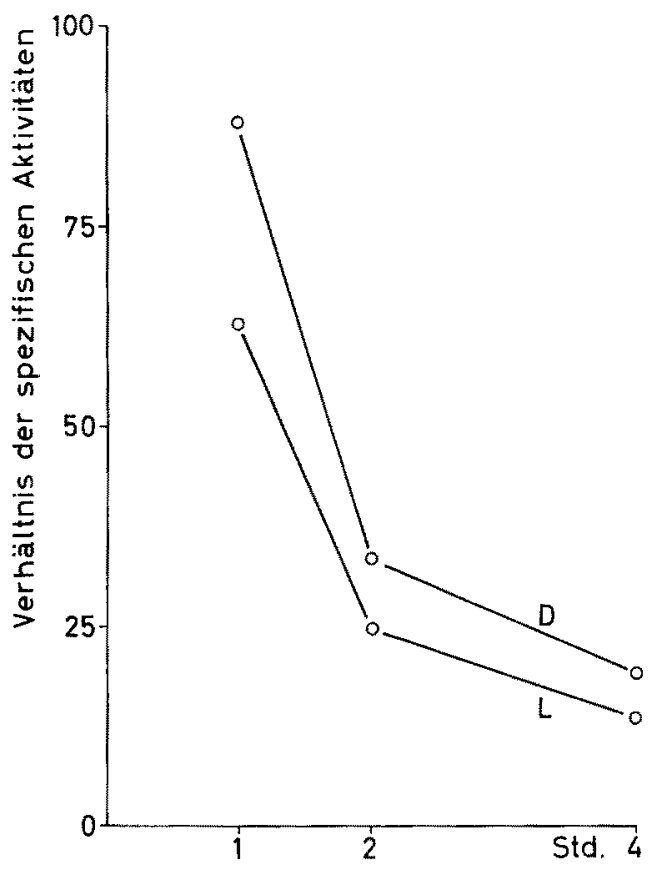

Abb. 3: Verhältnis der spezifischen Aktivitäten von leicht säurelöslichen Verbindungen und Phosphatiden in dunkelgehaltenen (D) und belichteten (L) Augen bei verschiedener Versuchsdauer. Mittelwerte aus 6 Versuchen pro Kurvenpunkt

Dies bedeutet, daß im Licht die Einbaurate in die Phosphatide unabhängig von der allgemeinen Stoffwechselerhöhung der Fliegenaugen - die sich in der Verstärkung des Gesamteinbaues ausdrückt - zusätzlich gesteigert ist.

Bei Belichtung erfolgt also ein spezifisch erhöhter Umsatz dieser Stoffe, die wesentlich am Aufbau der zellulären Membranen beteiligt sind. Dieser Befund ist ein starker Hinweis auf die Bedeutung, die die Phosphatide bei der Funktion des Auges, also bei der Erregungsbildung in den Lichtsinneszellen nach adäquater Reizung, haben.

\section{ZUSAMMENFASSUNG}

1. Bei Belichtung des Facettenauges von Calliphora finden sich keine Veränderungen in den Mengen der phosphathaltigen Substanzgruppen, die zu den sogenannten Baustoffen gehören.

2. Unter den zum energiebildenden Stoffwechsel gehörenden Metaboliten erfolgen bei Belichtung Veränderungen, die auf einen hohen Energiebedarf in den Augen hinweisen, insbesondere eine Verringerung des ATP und eine Zunahme des ADP und des freien anorganischen Phosphates.

3. Die Belichtung verursacht eine Erhöhung der Umsatzraten aller phosphathaltigen Verbindungen; diese ist auffallend stark in der Gruppe der Phosphatide. 
4. Da dieser besonders erhöhte Umsatz der Phosphatide einen spezifischen Effekt darstellt, wird auf eine spezielle Bedeutung der Phosphatide für die Funktion der Lichtsinneszellen geschlossen.

\section{ZITIERTE LITERATUR}

AKIYA, S., 1956. A study on determination of phosphorus metabolism in the retina by use of 32P. Acta Soc. ophthal. jap. 60, 336-343 (826-833).

Bergmeyer, H.-U., 1962. Methoden der enzymatischen Analyse. Verlag Chemie GmbH., Weinheim/Bergstraße, 1065 pp. (insbesondere p. $539 \mathrm{ff}$ u. p. $573 \mathrm{ff}$.).

Coren, L. H. \& Noenl, W. K., 1960. Glucose catabolism of rabbit retina before and after development of visual functions. J. Neurochem. 5, 253-276.

Honjo, J. \& Kato, M., 1952. Bildung und Ausscheidung der Phosphorsäure von der Froschretina. Seiro Seitai 5, 43-50.

- 1953. On the diffusion of ${ }^{22} \mathrm{p}$ from the rod outer segments. Annotnes zool.jap. 26, 186-191.

- 1955. Aufnahme des Radiophosphors durch die Froschretina. Z. vgl. Physiol. 37, 169-179.

Hoffmann, C. \& LANGer, H., 1961. Die spektrale Augenempfindlichkeit der Mutante chalky von Calliphora erythrocephala. Naturwissenschaften 48, 605.

Lange, H. \& Simon, M., 1922. Uber Phosphorsäureaussheidung der Netzhaut bei Belichtung. Hoppe-Seyl. Z. 120, 1-29.

Langer, H., 1960a. Über den Einfluß von Licht auf den Stoffwechsel des Auges von Calliphora erytbrocephala MkIG. Verh. dtsch. zool. Ges. 53, 226-234.

- 1960b. Die Wirkung von Licht auf den chemischen Grundaufbau des Auges von Calliphora erythrocephala Meic. J. Insect Pbysiol. 4, 283-303.

- 1962a. Untersuchungen über die Größe des Stoffwechsels isolierter Augen von Calliphora erytbrocephala MeIG. Biol. Zbl. 81, 691-720.

- 1962b. A new eye colour mutation in Calliphora erythrocephala Meig. Nature, Lond. 194, 111-112.

- \& PAtat, U., 1962. Uber die Bedeutung einer neuen Augenfarbmutante von Calliphora erythrocephala Me1G. für die Untersuchung der Funktion des Facettenauges. Verb. dtsch. zool. Ges. 55, 174-180.

Lohmann, K., 1928. Uber das Vorkommen und den Umsatz von Pyrophosphat in Zellen. I. Nachweis und Isolierung des Pyrophosphats. Biochem. Z. 202, 466-493.

Martn, J. B. \& Doty, D. M., 1949. Determination of inorganic phosphorus. Analyt. Chem., Wash. 21, 965-967.

Schneider, W. C., 1945. Phosphorus compounds in animal tissues. I. Extraction of desoxypentose nucleic acid and of pentose nucleic acid. J. biol. Chem. 161, 293-303.

SEKOGUTI, Y., 1953. On the fractionation of phosphorus compounds in the frog-retina. Seiro Seitai 5, 78-84.

Warburg, O., Posener, K. \& Negelein, E., 1924. Uber den Stoffwechsel der Carcinomzelle. Biochem. Z. 152, 309-344.

\section{Diskussion im Anschluß an den Vortrag LANGER}

Horstmann: Ich habe zwei kurze Fragen: 1. Wieviel Material wurde für einen Versuch verwendet? 2. Welche Phosphatide wurden gefunden?

LANGER: 1. Es wurden für eine Aufarbeitung nach SchneIDER die Augen von etwa 5 Fliegen eingesetzt, das Material reicht für Doppelbestimmungen in den Fraktionen. 2. Die Versuche zur Auftrennung der Phosphatidfraktion mittels Dünnschichtchromatographie werden zur Zeit durchgeführt. 
URICH: Wie groß ist der Prozentsatz des gesamten verfütterten Materials, das in die Retina inkorporiert wird, verglichen mit dem Anteil der Retina an der gesamten Körpermasse?

LANGER: Der Mengenanteil der beiden Augen am gesamten Lebendgewicht der männlichen Fliegen beträgt etwa $4 \%$. Der Gesamt-Einbau des ${ }^{32} \mathrm{P}$ ist - wahrscheinlich wegen der wechselnden Aufnahme vom Darm her - ziemlich verschieden. Im Einbaumaximum bei etwa $4 \mathrm{Std}$. nach der Fütterung finden sich 0,5 bis $1 \%$ der gesamten verfütterten Aktivität in den Augen wieder.

Locker: Haben Sie die P/O-Quotienten bestimmt?

LANGER: Der P/O-Quotient läßt sich hier nicht berechnen, da die Bedingungen der Og-Verbrauchsmessung (Autrum \& Tscharntke 1962; Z. vergl. Physiol. 45, 695-710) aus Gründen der Versuchsanordnung andere sein müssen als die bei den Phosphatumsatzmessungen, besonders im Hinblick auf die Belichtung. 